curability of the phthisis. To quote the mortality table with which I opened this essay:-

The mortality after aspiration is . . . . 87.5 per cent.

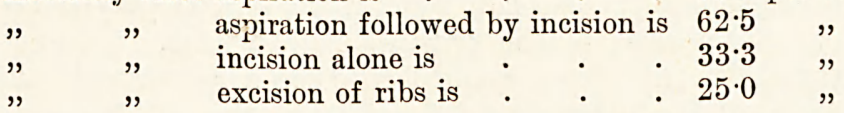

Taking together all the cases I have gathered in a previous decade, not before the days of antiseptic treatment of wounds, the mortality reaches 48.27 per cent., with which my 25 per cent. mortality, after excision of ribs, compares favourably.

I need not recapitulate the other points which I have dealt with throughout the paper, but I will refer to three points only. First, in cases where ribs are removed, a second operation is required in 10 per cent., but in cases where this is not adopted, a second operation is required in 66 per cent. Second, that in hospital cases treated by other treatment than by excision of ribs, the average stay of each patient in the institution is one hundred and eight days; it is lessened to sixty-eight days in cases treated by excision of ribs. Third, that in a straightforward case, without any complication, in a young adult or child, the average duration of the wound after operation is fifty-three days.

\title{
GALL STONES.
}

By Rutherford Morison, F.R.C.S., Surgeon to the Royal Infirmary, and Consulting Surgeon to the Dental Hospital, Newcastle-onTyne.

\section{Statistics. ${ }^{1}$}

Cholecystotomies, seven cases; all recovered.

Cholecystostomies, eight cases ; all recovered.

Cholecystectomy, one case ; recovered.

Choledochotomies, five cases; one recovered, four died.

Gall stones not operated on; two cases. died.

Of the twenty-one cases operated on, seventeen recovered and four

TeN years ago, in introducing a discussion on gall stones, ${ }^{2}$ I made some suggestions, based on general principles of surgical pathology, as to the causes of the symptoms and the physical conditions met with in cases of cholelithiasis. Time has only served to convince me of their truth and usefulness; and as they have not yet received the recognition which $I$ think they deserve, I intend in the present paper to restate them, together with my conclusions

${ }^{1}$ Statistics supplied by G. Grey Turner, Surgical Registrar, Royal Infirmary, Newcastle-on-Tyne, of cases of gall stones operated on by Mr. Morison during 1903 in the Royal Infirmary and in a private hospital at Newcastle-on-Tyne. Drawings by W. G. Richardson, F.R.C.S.

2 Jour. Northumberland and Durham Med. Soc., 1895, p. 21. 
based upon them, and to add any further observations that have since occurred to me.

"Gallstone attacks" are amongst the most painful of human ailments, and once they have occurred they are apt to be repeated at frequent intervals. On the contrary, gall stones may be present in the gall bladder for long periods without giving rise to any appreciable symptoms.

In searching for an explanation of these apparently contradictory facts, I remembered the behaviour of the urinary bladder in the presence of stone. Every surgeon knows that a large urinary stone which has taken years to form may be found with symptoms of only a few weeks' duration; and that, notwithstanding the presence of a stone, such marked amelioration of the bladder spasms and pain may follow relief of a coexisting cystitis, as to prove that the inflammation plays a very important part in the production of the characteristic symptoms. Like gallstone attacks, "attacks of gravel" are liable to recur; for in both they are due to cystitis, of which the stones are the predisposing cause, and the complete and permanent cure of the cystitis, so long as the irritating calculi are present, is improbable.

The terrible pains of gallstone attacks I attribute to the wellknown physiological qualities of unstriped muscle. Its forcible contraction causes the most excruciating pain, of which labour pains are the best known example.

The first facts noted were-

1. That gall stones may be present and cause no symptoms;

2. That once symptoms have occurred, they are likely to recur; and

3. That gallstone attacks are excruciatingly painful.

$\mathrm{My}$ interpretation of these facts is as follows:-

1. That in an uninflamed gall bladder, gall stones may lie undisturbed, and cause no symptoms.

2. That cholecystitis is the necessary irritant.

3. That when cholecystitis accompanies the presence of gall stones, the vigorous and repeated efforts of the gall bladder to expel its contents by forcible contraction of its unstriated muscular coat are the cause of the pains peculiar to gallstone attacks.

A few years later I learned, from a case in which I had excised the gall bladder, and in which gall stones recurred, that the characteristic pains might be present though the gall bladder was absent. When I met with this case, the fact that the common bile duct, like the gall bladder, possesses unstriated muscle in its walls, had hitherto escaped my attention.

Another fact noted was that serious results are scarcely at all due to the hundred more or less stones in the gall bladder, but to the one or more driven into the ducts. This is a point apt to be forgotten even now, judging by current literature, with its history 
of stones escaping after operation; stones which must have been left in the ducts, for in the gall bladder they can scarcely be overlooked.

The urinary bladder resembles the gall bladder more, perhaps, than any other hollow muscular-coated viscus, but all hollow viscera respond similarly to the stimuli of irritants. In the consideration of special diseases, sufficient importance is not, to my mind, attached to general surgical principles; even the details of these can be understood and explained if due consideration be given to the behaviour of the hollow viscera as a class. The fashion also of attributing every result, when inflammation is present, to the ravages of organisms, has hindered the progress of pathology almost as much as it has advanced it.

The first fact we learn from consideration of the behaviour of the gall bladder, the urinary bladder, the intestines, the vermiform appendix, the stomach, and the Fallopian tubes, is that violent contractions intended for the expulsion of their contents are accompanied by severe pain, and, when acting against a partial obstruction, these contractions are followed by hypertrophy of their walls, and, later, by diminution of their cavity.

The next fact is that, if they find the obstacle insurmountable, the muscular coats cease to contract, the violent pains abate, paresis of the muscular coat occurs, and is followed by passive distension of the viscus, and, later, degeneration of the unstriped muscle, and inflammatory changes in the viscus, may produce profound alterations in it. This explains hydrops of the gall bladder distended by its own secretion, the distended gall bladder of malignant pancreas, painless urinary retention with passive overflow, painless distension of the cæcum following obstruction to the sigmoid flexure, hydrops of the vermiform appendix, the enormous painless distension of the dilated stomach from obstructed pylorus, and hydrosalpinx following occlusion of the Fallopian tubes.

The next fact learnt is that, given complete obstruction with an active inflammation superadded, the intravisceral tension may become so acute that partial or total gangrene of the involved viscus, followed by its rupture, will occur. In the gall bladder such a gangrene appears first as a patch at the fundus, and gradually spreads, if the tension is not relieved by rupture, so as to involve the whole body of the gall bladder. That such a rupture of the gall bladder is not purely mechanical, is proved by the fact that the artificially distended gall bladder tears towards its neck. That the site of gangrene is determined by the vascular supply, is made evident by consideration of what happens in the urinary bladder, the appendix, and the cæcum. In a young man, æt. 25 , admitted to the Royal Infirmary, Newcastle, 20 th December 1888, suffering from retention of urine of four days' duration, due to a gonorrhœal prostatic abscess and excessive 
whisky drinking, I found his peritoneal cavity distended with urine, which had escaped through "a small opening scarcely as large as a split pea, of grey ashy colour, at the superior portion of the posterior wall of the bladder" (i.e. the portion furthest from the main supplying arterial trunks). Gangrene in the inflamed and distended appendix, strictured near the cæcum, commences on the convexity and near the tip (a point furthest from the vascular supply). Gangrene of the distended cæcum commences by small round patches in the neighbourhood of the anterior longitudinal' band (furthest from the vascular supply). Acute perforating ulcers of the stomach are found in a line midway between the greater and lesser curvature, i.e. furthest from the vascular supply.

A special note is necessary to explain Dr. Drummond's and my own view as to acute perforating gastric ulcer. It is that spasm of the pylorus often causes acute stomach distension of a temporary character. In one of these attacks the ischæmic patch resulting is so seriously damaged that gangrene of it follows. In the course of a few days, separation of the gangrenous patch results in perforation.

In all these instances I regard the gangrene as resulting from tension. The tension in all is mainly due to inflammation. But this is not, as is now generally taught, an invariable rule. For example, in a case of total gangrene of the cæcum following prolonged and complete obstruction due to malignant stricture of the sigmoid flexure, I found neither clinical nor pathological evidence of inflammatory phenomena. It is not true that gangrene always results directly from the attack of virulent micro-organisms. There can be no doubt that some rare forms of gangrene primarily result from infection by specially virulent organisms; but in the common variety of gangrene I shall continue to believe, till stronger evidence to the contrary has been offered, that interference with the vascular supply is the primary cause, and that organismal infection plays a secondary part. In other words, it is not until the tissues have been weakened by interference with their vascular supply that they afford a fit soil for such infection.

When I was a student, the greatest importance was attached to the presence of cystitis when operating for vesical stone. Crushing had to be done very gently, very little at a time; and the cystitis received every possible attention.

Gallstone surgery is in the same stage to-day; all the authorities engage chiefly in discussing the causes, the progress, the consequences, and the treatment of the inflammation, and neglect the stones. In the case of the urinary bladder, I recall the shock the surgical world received when Bigelow of Boston announced $^{1}$ that if the stone or stones were removed, the cystitis

${ }^{1}$ Amer. Journ. Med. Sc., Philadelphia, January 1878. 
would promptly get well. How long will it take surgeons to recognise that the same is true of the gall bladder? In both, exceptions occur to the rule, but they are rare.

Inflammation of the gall bladder differs in no respect from inflammation elsewhere. The presence of stones is a predisposing cause, which favours the development of inflammation. Cholecystitis not accompanied by the presence of stones is rare. It occurs, however, in typhoid fever and other intestinal infections, and occasionally a blood infection may cause it. The exciting cause of all serious forms is infection by a pyogenic micro-organism; the possible terminations of all are by-(1) resolution, (2) fibroid thickening, (3) partial destruction (ulceration and sloughing), (4) total destruction (gangrene). To describe the possible terminations of inflammation as distinct clinical varieties under such names as catarrhal, phlegmonous, and gangrenous cholecystitis, is to add to the difficulty of clear thinking, and to bar progress. To show the importance of grouping together similar structures, in order to understand pathological possibilities, I may mention that though I had not read of, or heard of, one elsewhere, I was for a long time on the outlook for a sacculated gall bladder, and eventually found one. Sacculation of the urinary bladder is well known. Sacculation of the intestines, especially of the colon, had been recognised. I have drawn attention to the fact that similar changes are found in the gall bladder, the vermiform appendix, and the Fallopian tubes. The exact pathology of all is obscure; but it seems probable that they will all be found to have, as in the case of the urinary bladder, some relation to inflammation of the walls, and partial obstruction of the outlet.

The biliary ducts compare in many points with two other small muscular tubes, whose behaviour is known, the ureter and the urethra. All of them, when irritated by the passage of a foreign body, are likely to contract spasmodically on the intruder, so that a small stone may temporarily form a complete obstruction. At a later stage the walls of the muscular tube recede from the foreign body, and active dilatation follows. Intermittent jaundice and attacks of temporary hydronephrosis may be produced respectively by a stone lying in the bile duct or in the ureter.

With these general facts as guides, the diagnosis and prognosis of gallstone cases is made easier.

Diagnosis.- It has already been stated, and indeed the fact is generally admitted, that uncomplicated gall stones may lie in the gall bladder and produce no symptoms. Their diagnosis then is not likely to be possible, nor is it of practical importance. The earliest recognisable symptom of gall stones, and one consequently of great importance, is usually described in popular language as an "attack of spasms," and the "attack" is generally referred to 
the stomach. ${ }^{1}$ The sudden and severe character of the pain are its chief features. Often the pain can be fairly localised over the position of the gall bladder, and, after it has passed off, it invariably leaves marked tenderness on pressure over the gall bladder.

Such an attack may produce no change in the anatomical position of the stones, or it may drive one or more into the neck of the gall bladder, or through it into the cystic or common ducts, through which it may escape into the duodenum, to be passed in a few days with the fæces. A large number of small stones are not infrequently passed in this way.

The diagnosis in these cases is based upon the history of "spasms," and upon the tender spot, found by pressing the finger tip over the gall bladder at a point at the outer edge of the right rectus muscle, opposite the ninth costal cartilage. Other conditions, producing the same symptoms, are pyloric ulcer and appendicitis, if the vermiform appendix lies under the liver, as it does when the cæcum has failed to descend. I know of no means of distinguishing these from attacks due to gall stones, and have recently, with a full knowledge of the possibilities, operated for gall stones, to find a pyloric ulcer and an inflamed subhepatic appendix. The same uncertainty of diagnosis will be felt in all cases when reliance has to be placed on the history, and physical signs are absent or of doubtful value.

If stones are passed into the duodenum, a history that shivering and vomiting occurred, and that jaundice followed after some of the attacks, will probably be obtainable.

Pancreatitis in some cases so closely simulates gallstone attacks as to make diagnosis impossible. In many of these cases the intermission of the pains and jaundice can only be explained by the view that a temporary increase of the pancreatic swelling causes for a time obstruction of the common duct, and this explains the fact, which I have not previously seen noted, that the gall bladder may be thick-walled and contracted, whereas when the obstruction is complete, as in cases of pancreatic tumour, the gall bladder is thin-walled and distended, and the jaundice permanent. In a case recently operated upon, the history of a sudden commencement with agonising pain, followed by jaundice, and repeated similar attacks, led me to make a diagnosis of gall stones and pancreatitis, because I was able to feel definitely the hard, nodular, enlarged, and tender pancreas; but operation showed that there were no gall stones, and no signs, such as adhesions or thickened ducts, that there had been any. There was only a pancreatitis, the cause of which was not discovered. The size and condition of the gall bladder may be of considerable diagnostic value in these pancreatic cases, for it is impossible from examination of the hard, enlarged, nodular pancreas to say whether the infiltration is due to malignant or inflammatory

${ }^{1}$ Loc. cit. 
deposit. A small gall bladder favours the diagnosis of simple, a large one that of malignant change in the pancreas.

If a large stone is firmly driven into and completely blocks the neck of the gall bladder, or the cystic duct, the attack is attended, as a rule, by unusually severe and long-continued pain, which only gradually abates, as distension-paralysis of the muscular coat of the gall bladder develops. Sooner or later after this attack, the swollen tense gall bladder will be discovered as a tumour below the ninth costal cartilage, in a line between that point and the umbilicus.

This tumour has been mistaken for many other conditions, but the chief error made is in confounding it with a movable kidney, about which much mischievous nonsense has been written, for it is very seldom the cause of serious symptoms.

The chief diagnostic points are-That movable kidney is most often found in thin, nervous women before forty, and that the health is not seriously impaired. The swelling is not easily felt with one hand on the anterior abdominal wall, and patient breathing quietly. Pressure on the swelling produces sensation characteristically disagreeable. The swelling slips back into the kidney region easily and completely, and remains there when the patient is recumbent, unless disturbed by bodily or respiratory movements. It is freely movable in every direction.

A tumour of the gall bladder is most often found in fat women after forty, and there is a history of loss of flesh and strength. The tumour is tender when pressed upon; is felt close to the anterior abdominal wall, and moves with respiration. It has to be coaxed into kidney region; it never disappears, and will not remain there unless artificially retained. It is moored at its upper part.

On bimanual palpation, in the case of movable kidney, the swelling is flattened, solid, but not hard. The swelling is jerked down by forced inspiration; it is easily detained by the hands pressed together above or below it, and it is apt to jerk suddenly back again when the pressure is relaxed. The loin feels hollow when the swelling is in the abdomen.

The distended gall bladder is rounded; it feels very hard, because it is tensely distended with fluid; the tumour descends steadily and aggressively on forced inspiration, and it ascends vigorously on expiration against any obstacle. The kidney may be felt behind, and independently of the tumour.

On percussion, there is a tympanitic note over the renal swelling, while in distended gall bladder the note is dull over the tumour, and the dulness is continuous with that of liver.

The tumour, as I have said, may be discovered sooner or later after the attack, and in some cases may be the seat of most acute and dangerous mischief; whilst in others the trouble it occasions is so slight, and the changes in it are of such a chronic 
character, that it may only be discovered accidentally in the course of a complete examination. Midway between the severe and the mild cases there is a third type, less acute than the first, but more serious than the second, attended by purulent infection of the contents of the distended gall bladder.

In the most severe type, a few hours after the commencement of the illness; the swollen, very tender, and tense gall bladder may be felt as a hard tumour, and the patient looks very ill. The temperature and pulse are both quickened, vomiting and thirst are present, the vital powers are much depressed, and acute

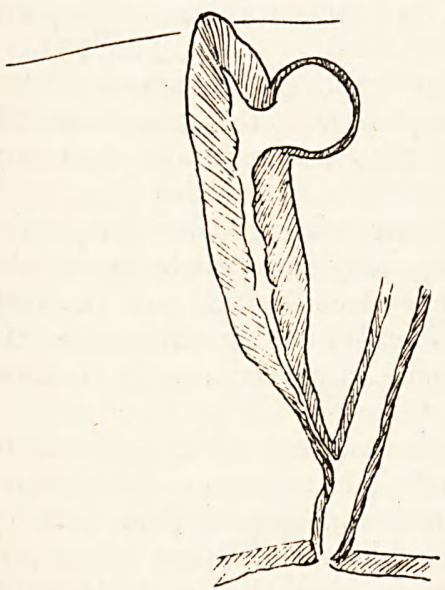

Fig. 1.-Sacculus of gall bladder, from old gallstone case.

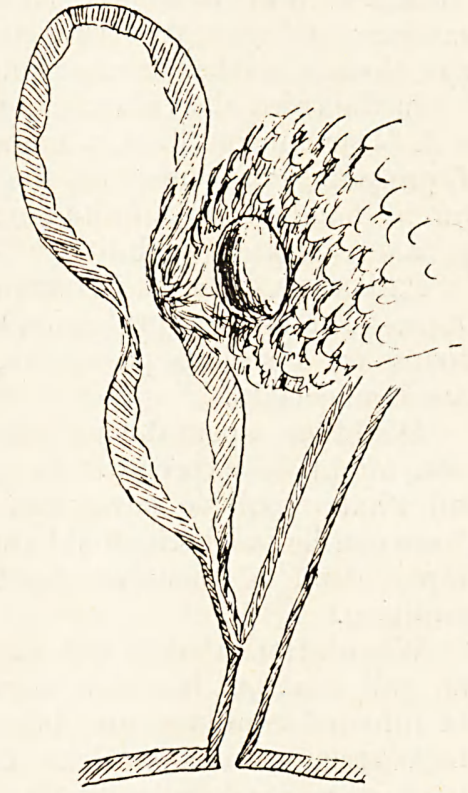

FiG. 2.-Perforated gall bladder, with omentum, in which a gall stone has become encysted, blocking the perforation. The gall bladder was very thick, and of hour-glass shape.

pneumonia is a frequent complication. The general condition is that produced by acute septicæmia; the local condition one of gangrene of the gall bladder. There can be little hesitation in attributing the severity of the attack to the presence in the gall bladder of virulent organisms, favoured in their growth by conditions not yet understood. One of these conditions I believe to be the presence of a quantity of fluid bile in the gall bladder, which I have always found during operation on these cases. It appears to me probable that a gall bladder filled with bile, when impaction of a stone occurs, is more likely to form a suitable incubator for germs, and to develop acute mischief, than one 
which has been thoroughly emptied by contraction before complete impaction of the stone occurred. In this case the gall bladder becomes distended by its own secretion, which probably, like other mucous secretions, contains some antiseptic principle, and this may account for the frequent absence of suppuration of its contents.

In the second class of case, the distended gall bladder contains pus. The diagnosis is based chiefly upon the tenderness of the tumour, which never disappears; on its becoming more indefinite in outline from omentum adhering round it; from increasing uneasiness and weakness, though these may not be marked features ; and upon recurring rigors, night temperature and sweatings, though neither is invariable.

In the third class of case, in which the gall bladder is distended with clear mucoid fluid, a diagnosis may be based on the history of preceding "spasms," on the freedom with which the swelling can be handled, its definite outline, its position and relations, and its free respiratory mobility.

Prognosis of Gall Stones in the Gall Bladder and Cystic DUCT.-The medical profession has been slow to recognise that gall stones are a serious possession, and that the prognosis in every case is uncertain.

Doubtless spontaneous cures occur with greater frequency than might be expected. It is surprising how ducts can dilate, and what expulsive power can be exercised by the gall bladder. There can be but little doubt that the gall bladder can completely empty itself of moderate-sized stones, and recover its normal condition.

When attacks recur but a stone is not impacted, the wall of the gall bladder becomes thickened and its cavity contracted. ${ }^{1}$ Its infected contents are bile-stained inspissated fluid, pus, or thick stringy mucopurulent fluid, which sometimes glues the stones together. Inflammation spreads from the cavity through the walls to the surrounding peritoneum, and the gall bladder becomes enveloped in an indurated vascular mass of omentum. The fixation so produced may lead to a natural cure, with fibroid thickening and degeneration of the coats of the gall bladder and fixation of the stones, if the cystic duct is sufficiently patulous to allow of efficient drainage. I have many times, in the postmortem room, found such a gall bladder sometimes dried up, and, in whole or in part, calcareous, grasping its calculous contents. If the cystic duct does not admit of effective drainage, an abscess forms in and around the gall bladder, and the prognosis is that of localised gall bladder suppuration. In the most acute cases the prognosis is that of septic peritonitis, resulting from perforation of a viscus. Such instances are rare, for the reason that previous

${ }^{1}$ Sections of such gall bladders, made for me by Dr. Bolam, show that the thickening is due chiefly to an increase of the muscular coat. 
attacks have generally left adhesions which localise the escaping septic contents; though more than once, when operating for acute peritonitis in an abdominal emergency, I have found the peritoneal cavity distended with bile-stained purulent fluid, a gangrenous ruptured gall bladder, and gall stones free in the abdomen. The only hope for such cases is immediate operation.

The prognosis is serious if there be marked tenderness of the gall bladder, loss of flesh and strength, or elevation of temperature or recurrent rigors, which suggest suppuration in the distended gall bladder. As a rule, such a gall bladder is surrounded and protected by the omentum, which possesses this function in so remarkable a degree, that I have for some years spoken of it to students as the "abdominal policeman." If there are no adhesions, the pus may rupture into the general peritoneal cavity. The more common termination is by a localised abscess round the gall bladder. This may follow several courses, some of them tending to recovery, others to death.

Rupture of the gall bladder, as I have elsewhere pointed out, is not a rare termination of suppuration of its contents, and I have satisfied myself that the contents can be absorbed, the stones encysted, and the patient get well. On several occasions, when operating, I have found stones lying outside of the gall bladder, safely wrapped up in omentum, with or without purulent surroundings, and a plug of omentum securely glueing up a hole in the gall bladder.

The abscess, as is well known, may leak into an adjoining viscus, duodenum, stomach, and colon, to the relief of all urgent symptoms; but possible intestinal obstruction from gall stones or future gall bladder trouble, after closure of the fistula, have to be remembered in giving the prognosis.

Occasionally the abscess may open externally through the abdominal wall; more rarely pus finds its way through the gall bladder into the liver substance, forming a hepatic abscess.

When the gall bladder is distended by a clear mucoid fluid, as suggested by a large, hard, freely movable tumour, without markedly active symptoms, the prognosis depends upon whether the block is fixed or temporary, and what further changes occur in the gall bladder contents. The most probable is pus infection, and the prognosis is then that of the previous variety. The tumour may, however, continue for years unchanged, or it may steadily increase till it forms a large swelling, or it may gradually shrink and finally disappear. If a small stone is impacted in the cystic duct, the gall bladder is distended on some days and not palpable on others, when the absence of spasm allows escape of the gall bladder contents. If the stone is not firmly impacted, it may fall back into the gall bladder, when its contractions cease. This condition frequently occurs when a single good-sized stone is present. I have had the opportunity 
of observing instances of each of these terminations, and in one case, when I aspirated the gall bladder on two separate occasions, ten years after the last aspiration, I was able to feel a very hard symptomless and painless swelling, which I believed was the atrophied gall bladder contracted on a large stone.

It will thus be seen that, except in the most acute attacks, which are very rare, recovery unaided by operation is possible in these cases. Indeed, some medical men of large knowledge and experience have told me that patients rarely died from gall stones, and that operations of which they had knowledge were not so successful as they had been led, by perusual of the most recent literature, to expect. Considering the serious nature of the attacks, the grave illness which often follows, and the frequency with which recovery occurs, more often, I believe, than in any illness of like severity, a too optimistic belief in the probability of recovery, based upon the remembrance of two or three wellremembered and apparently hopeless cases which got well without operation, has been formed. Surgical experience has abundantly proved that gall stones are occasionally a grave danger to life, and frequently a cause of chronic and serious ill-health. I can recall a considerable number of deaths from gall stones for which no operation had been performed, and post-mortem examinations occasionally reveal the fact that gall stones are a dangerous possession. The illness resulting from them is directly or indirectly the cause of premature death much more frequently than is suspected.

If a stone is driven into the common duct and arrested at any part of its course, the same spasmodic pains are present as in the previously described condition. Shivering and vomiting are likely to be more pronounced symptoms. The escape of bile is prevented, and the gall bladder becomes tense. If previous attacks have not left it small, contracted, and bound down by adhesions, it may be felt in the usual position as a tense, tender, rounded swelling; but this is comparatively rare. As a rule, the thickening and contraction left by hypertrophy and inflammation resulting from previous attacks prevents the gall bladder from distending sufficiently to form a tumour, hence the aphorism that " jaundice with a gall bladder tumour is not the result of gall stones." Like the majority of aphorisms, there are exceptions to this one. A gall bladder which has once been much dilated probably never recovers its previous size. Jaundice follows the attack in a few hours, and may pass off in the course of a day or two or never totally disappear. In cases where it does not totally disappear there is a recurrence of the attacks, and with each recurrence an increase of the jaundice.

Diagnosis.- In typical instances the diagnosis of gall stone in the common duct is easy. It is unlikely that an illness commencing with a sudden violent pain in the upper abdomen, followed 
by recurrences of the pain and intermittent jaundice without a palpable distension tumour of the gall bladder, is due to any other cause. The diagnosis may be made certain by the discovery of gall stones in the fæces, and these should always be carefully sought for several days after the attack. The behaviour of the duct in the presence of a stone must be considered before going further, as on this depend many of our conclusions.

A small stone, one not larger than a pea, will cause temporary occlusion of the common duct and jaundice: a large stone, one the size of a walnut, will not cause its permanent occlusion; the jaundice is intermittent. Here it must be noted that in some rare cases the symptoms and signs of gallstone closely simulate those of malarial fever. Repeated shivering attacks without pain, attended by fever and followed by jaundice, may be the only evidence of gall stones in the common duct. In these instances I have always found the gall bladder contracted and functionless and the common duct largely dilated. The painful attack during which the stone was driven into the duct may have occurred so long ago as to have been forgotten. How are such facts to be explained?

The conduct of the urethra, ureter, and intestine, similar muscular tubes whose action may be more readily observed, offers a solution of the difficulty. A small stone in the ureter will cause a temporary obstruction, followed by dilatation above the site of the obstacle, and by hydronephrosis of the corresponding kidney. These attacks will recur from time to time so long as the stone remains. A bougie introduced into the urethra will at times be grasped so tightly that its onward progress or attempted removal is strongly opposed. The same bougie, tied in, will cause dilatation and allow of the easy passage of a large instrument. When a large gall stone escapes into the intestine, symptoms of acute intestinal obstruction may follow; but, after a time, passage of flatus and fæces occur, with relief to the symptoms; and it rarely happens that a patient dies until worn out by repeated attacks due to the single stone. In each instance the presence of a foreign body causes spasm of the muscular tube, the intruder is firmly gripped, the lumen is completely occluded, acute obstruction supervenes, and its characteristic signs and symptoms follow. When a gall stone is driven into the common duct, it causes spasm of the duct and complete occlusion of it, followed by obstruction to the escape of bile into the duodenum, and jaundice. After a time, the spasm relaxes, bile again passes, the jaundice and other symptoms tend to disappear; and even though the stone remains, there may be no evidence of its presence. The capacity of this small tube for expansion appears to be almost infinite, for I have on several occasions seen it so much distended as to be mistaken for the duodenum. In these circumstances the duct may become paretic, hence the absence of pain previously noted. In this con- 
dition, an ordinary stone lies loose in it, and such stones have been described as "floating." Two explanations may be offered for the recurrent attacks and jaundice in these cases, where only a single stone exists in the common duct. The first, and this, I believe, from observation on operation cases, to be the usual one, is, that spasm of the duct is readily produced by stimuli, e.g., a hearty or indigestible meal, exercise, etc.; the second, that a "floating" stone may be suddenly displaced and may temporarily block the duodenal orifice of the duct.

Prognosis.--In many instances, as has been previously stated, a stone or stones will make a successful passage through the ducts into the duodenum and escape with the fæces. In this manner, cure may be effected by natural resources. In at least one instance I had definite proof of this possibility. For more than a year a stout middle-aged woman had been incapacitated by frequently recurring attacks of gallstone colic, and after the majority of attacks a stone was recovered from the fæces. Of these calculi she showed me a considerable collection. The last attack a week before had been more prolonged and more severe than any of the others, and she determined to have an operation. I found the gall bladder thick-walled and contracted, with some adhesions round it, but empty. All the palpable ducts were thickened and enlarged, but empty; and it was evident that all the stones had been passed. This view has been confirmed by the fact that she had no attack since the operation, several years ago.

But unfortunately the stones do not usually escape. The opening of the common duct into the duodenum is its narrowest and most rigid portion, and a stone which may readily enough pass down the rest of the duct is unlikely to be able to pass this. The arrest of the stone is followed by dilatation of the duct, the gall bladder may empty itself into the duct, and the stone is carried back by the forcible bile stream into a higher portion of the duct. There it may lie for long periods and offer no sign of its presence, as the duct is dilated round it sufficiently to allow of the free escape of bile. In one of my acute cases, after operation and removal of several stones from the common duct, and of a large number from the gall bladder (which was completely emptied), the patient was "cured" for more than a year. She then had a return of such urgent symptoms that a second operation was necessary. This disclosed a single hard stone in the common duct, the size of a large filbert, with facets upon it. Since these facets must have been produced by the pressure of other stones, it had obviously been in the ducts all this time without producing any symptoms, as the recovery appeared to be perfect. Since its removal several years ago, the patient has remained well.

The common course of events is, that the irritation of a stone in the duct is the precursor of inflammation, and the swelling and obstruction of the ducts caused by this, hinders the escape of bile 
sufficiently to prevent the entire disappearance of jaundice. The usual history in these cases is of recurrent attacks of pain, due to spasm of the ducts caused by the irritation of the stone in the inflamed duct, or else due to the escape of fresh stones from the gall bladder into the ducts. The painful attacks are next followed by an increase of the jaundice, and are accompanied by vomiting, shivering, and rise of temperature. These attacks are so typical that they are universally recognised as characteristic of the presence of gallstone in the common duct, and they occur in the overwhelming majority of cases. It is possible to go further, and in these cases of sudden severe pain, followed by persistent jaundice with intermittent pains and increase of jaundice, rigors, and rise in temperature, to make a diagnosis of gall stones and infection of the bile ducts.

It is the latter which requires most serious attention when the prognosis comes to be considered. The inflammation of the ducts, like other septic infections, is always uncertain in its course and terminations, and from a mild commencement may develop a furious end. It seems probable that if the infected bile has a free outlet into the duodenum, no serious symptoms are likely to occur; but if the pent-up bile is retained in the ducts, the general symptoms of septic infection become pronounced, and the local changes consequent on inflammation occur in the ducts and surrounding liver. These are, it is scarcely needful to say again-(1) resolution, (2) fibroid thickening (in liver cirrhosis), (3) partial destruction (in ducts-ulceration, in liver-abscess), (4) total destruction-gangrene in ducts.

Serious though these conditions are, it is possible to have spontaneous recovery from them all. This is generally brought about by the formation of a fistulous communication between the duodenum and the common duct or gall bladder. The stone causes ulceration, a localised abscess (prevented from becoming diffuse by previous adhesions) opens into the intestine and allows of the escape of the stone; a fistulous communication permitting of free bile drainage is established; and, notwithstanding the fact that many stones may be left, a "symptomatic cure" is brought about. More rarely, a suppurating gall bladder adheres to the abdominal wall and ruptures there, leaving a biliary fistula, but removing all serious symptoms. Still more rarely, stones escapc by ulceration through the duct, leaving this channel free, into a cavity limited by adhesions; and become encysted there, with subsequent closure of the opening in the duct, and it may be with or without the formation of pus. If the pus escapes into the intestine, healing of the abscess may occur, leaving the stones buried in adhesions.

But the dangers in such cases are, that the patient is worn out by the recurring attacks of pain, by septic absorption from the inflamed ducts, gall bladder, and liver, and by the toxæmia 
resulting from bile absorption. Sudden death, apparently from heart failure, is no uncommon event in such cases, and may occur during what is considered to be convalescence. In such circumstances one of my patients died suddenly whilst on the commode; a second in the act of getting out of bed; a third complained whilst convalescent of a sudden severe return of pain, and died in a few moments; on post-mortem examination, I found a small stone impacted in the duodenal orifice of the common duct. Operations on such patients prove that their illness has increased their susceptibility to all the three great dangers of operations, namely, sepsis, hæmorrhage, and shock.

A second, though rare cause of sudden death, was illustrated in the case of a stout woman whom I had seen many times with recurring gallstone attacks and jaundice. She appeared to be improving at the time of her sudden death. Post-mortem examination showed that death was due to a large intraperitoneal hæmorrhage which had come from the portal vein. A stone lying in the common duct projected through an ulcerated opening into the vein, where it lies in close proximity to the duct in the right border of the gastro-hepatic omentum.

Treatment.-Here the first consideration has to be given to surgical methods, and it is only when for some sufficient reason the removal of the stones cannot be advised that any other measures should be suggested. If a stone be discovered in the urinary bladder, no medical man would suggest that it should be left there till cystitis and kidney infection made the patient seriously ill, and its removal imperative; but this is a too frequent practice in the case of gall stones. Without doubting the diagnosis, some physicians will, after the failure of ordinary measures, recommend a patient suffering from gall stones to try a visit to Carlsbad! It is difficult for surgeons who know with how little risk stones can be removed from the gall bladder, to speak of such advice with proper professional courtesy. The risk of gallstone operations is the result of delay. Stones may be removed from the gall bladder with as little risk as is incurred in any ordinary surgical operation; and no hesitation should be felt in advising that operation be undertaken, before they have made their escape from that viscus, and with the object of preventing its occurrence. The record of the post-mortem examination of my cases shows more plainly than anything I can urge, the increased risks of operation postponed till stones have escaped into the common duct.

CASE 1.-Acute sepsis ; advanced liver cirrhosis.-All ducts throughout much dilated.

CASE 2.- Operation recommended five years before; œdema of all parts in the neighbourhood of liver noticed at operation. Liver ducts much dilated throughout: common duct size of duodenum; active 
septic infection with abscess in liver pouch and over liver caused death on ninth day after operation.

CASE 3.-Death forty-eight hours after operation from acute sepsis? Wound showed some signs of infection. Peritonitis limited to liver pouch. Whole of liver ducts dilated and containing purulent bilestained fluid. From the lesser ones almost pure pus escaped.

CASE 4.-Operation advised three years before. Chronic jaundice ; conmon duct size of duodenum. Many stones removed from common and hepatic ducts and one from gall bladder. Death forty-eight hours later. Acute sepsis?

Post-mortem.-Wound looked healthy; no peritonitis ; all bile ducts much dilated; innumerable gall stones had been left. In the very smallest intrahepatic ducts, gall stones varying in size from a pea to coarse sand were found.

Though it must be admitted that the results recorded here are exceptionally bad, they may serve the good purpose of emphasising what has been my universal experience,- - that operations on the gall bladder may be undertaken with confident assurance of success, but that the operation for the removal of stones from the bile ducts is a dangerous and often impossible one. Sepsis, the greatest danger of operations, is already present, and operation may be the means of increasing it; hæmorrhage, the next risk of operation, is more difficult to arrest and more likely to occur in jaundiced patients; and shock is readily produced and difficult to combat in patients who have suffered from recurrent gallstone attacks. Hence the high mortality. If any doubt as to the propriety of operation in gallstone cases is to be entertained, let it be kept for those worn-out jaundiced patients in whose common ducts stones have been retained for months or years, to whom operation has been only suggested as " a last resource," and in whom, even if immediately successful, the operation may fail to cure because all stones are irremoveable.

The Operation.-The abdomen may be opened by a variety of incisions, and that advocated by so great an authority as Mayo Robson, and mostly in favour with surgeons, is a vertical one through the right rectus muscle. The next favourite is an oblique incision parallel to and a finger's breadth below the right costal margin.

My own is a transverse incision in a line from the right border of the rectus muscle to the outer margin of the flank, and opens up the space between the liver above and the transverse colon below. I described this space in the Brit. Med. Journ., London, November 3,1894 , and there advanced what I considered, and still think, were good reasons why this operation was better than others. Special care must, especially in jaundiced cases, be taken to secure every bleeding point. It is certain, whatever may be the value of other measures to arrest hæmorrhage, that the ligature is still the best and the safest. In dividing the deeper 
structures, it is wise to pick them up in the forceps, draw them forward, and divide the tissues between ; for in this way the liver will escape incision, to which it is liable, from its resemblance to the muscular substance covering it.

When the gall bladder is distended, it often comes into view at once, and always does so on drawing the liver up and pushing the transverse colon down. A large flat sponge is next pushed into the hepatic pouch, to keep the colon down and to catch any escaping gall bladder contents. The gall bladder may be so large and so movable that its fundus is readily tilted out through the opening, and the operation is then very simple. After opening the gall bladder, the fluid contents escape. In many cases it will be observed that the first discharge is the clear mucoid secretion of the gall bladder, while towards the last the discharge becomes purulent, and finally consists of pus. The pus has sunk down to the neck of the gall bladder, just as the pus in purulent urine standing in a conical glass will sink to the bottom. When the gall bladder has been emptied, the stones are to be removed. This is most readily done by introducing the fingers of one hand underneath the gall bladder, and by pressure outwards between the fingers below and the liver above forcing the stones up to and through the gall bladder opening. When any difficulty is met with, a finger should be introduced into the gall bladder, and with this as a guide to the position of the stone, a scoop or forceps may help its escape. It must be remembered that the loose stones are not the cause of the trouble; and careful search is to be made for one that is impacted, either in the neck of the gall bladder or in the cystic duct. It is true that a collection of stones glued together by sticky gall bladder contents may have caused the obstruction, but this is rare. As a rule, a single large stone has become impacted in the neck of the gall bladder. Probes or finger inside of the gall bladder may miss this. It is important to feel the neck of the gall bladder, and indeed all the ducts, between the finger and thumb, introduced into the hepatic pouch and the foramen of Winslow. Between the finger and thumb so introduced, a stone in the neck of the gall bladder or the cystic duct can generally be squeezed back into the gall bladder and removed.

Having satisfactorily emptied the gall bladder, the next consideration is, How is it to be treated? And as this question receives a different answer from nearly all authorities, I will state my own practice, and the reasons for it.

Let it be assumed that the wall of the gall bladder is healthy, that the contents, although purulent, mean no grave infection, and that all stones have been removed.

The question now to be answered concerns the patency of the cystic duct. Probes are unsatisfactory instruments for the determination of this, for the cystic duct is a difficult channel to 
navigate. If bile flows into the gall bladder when the stone is removed, the patency of the duct is assured; if not, I pump the gall bladder with warm saline fluid, and am guided by its escape through the ducts or otherwise as to the patency or obstruction of the cystic duct.

With a patent cystic duct, I entirely close the gall bladder and return it to the abdomen. At the same time it is my practice to drain the hepatic pouch at its most dependent part by a tube for a few days, lest leakage should occur. I feel safer in leaving a tube in the hepatic pouch for a few days, because in one of my cases some bile did escape from the gall bladder six days after operation, and the presence of a tube prolongs healing for a few days only.

In these cases I expect the cholecystitis to cease after the removal of the stones, for the same reason that a urinary bladder cystitis is likely to disappear after the removal of a stone; and it seems to me to be as unreasonable to demand artificial drainage of the bladder in the one as in the other.

That operation for the complete closure of the gall bladder is safe, I know. I have no evidence that its ultimate results are other than satisfactory, though I have done it very many times and for several years, and patients so treated are able to leave hospital at the end of a week.

If the cystic duct is obstructed, the illness is recent, and the cause therefore likely to be temporary swelling. I drain the gall bladder by tying in a drainage tube and bringing it out through the loin, never by suturing the gall bladder to the parietes. Since adopting this practice more than ten years ago, I have never had trouble with a biliary fistula. I drain the gall bladder in the same way if there are signs, such as thickening, reddening, or presence of surrounding adhesions, that its walls are deeply infected.

If the gall bladder is acutely infected, with a patch or patches of gangrene which first appear at the fundus, it is usually found embedded in and adherent to a thick œedematous mass of omentum, from which it can be readily separated. It is tempting to think of removing the septic focus by excision of the gall bladder, a plan which has received the advocacy of Moynihan, but further experience is needed before I can accept his view. It is impossible in the most acute of these cases to remove the whole of the septic infection; for it has already passed beyond the confines of the gall bladder. Experience has taught me that extensive operations are badly borne by acutely septic patients, and the raw surface left by an extensive operation may allow of fresh absorption and increase of the septicæmia. I am content, as yet, to open, empty, and drain the gall bladder with a tube, and to protect and drain the hepatic pouch with a thick gauze strand. Let me also here point out that many of the arguments that are 
used in favour of cholecystectomy as a routine practice do not appeal to me. Though more than ten years ago I advocated removal of the gall bladder to prevent the re-formation of stones and to diminish the risk of biliary fistula, I have since become more conservative, for the following reasons: because a patient whose gall bladder I had excised seven years previously had a recurrence of gall stones in his common duct; because, after ceasing to attach the gall bladder to the parietes, I have never seen a biliary or other fistula remain; because I do not believe that the gall bladder will fail to recover its functions when emptied of stones; and because of its possible future uses.

I excise the gall bladder under the following circumstances: when it is the seat of malignant disease; if the cystic duct is occluded, and the occlusion is probably permanent; when a thickened diseased gall bladder has been separated from the intestine in a case of biliary fistula, in order to give the intestinal opening a better chance of closing; and when a stone in the cystic duct causes more than usual trouble to extract, the object being to remove the cystic duct and the stone together.

Choledochotomy.-The removal of a stone from the common duct may be easy, or may be attended by serious difficulties. In a thin, lax patient, whose hepatic pouch is free from dense adhesions, and with a stone in the common duct above the pancreas, the operation can be readily accomplished. With the forefinger in the foramen of Winslow behind the duct, and the thumb on the outside of the duct, a stone can be palpated, made prominent in a suitable position through the wall of the duct, cut down upon safely with the prominent stone as a guide, and squeezed through the incision in the duct. I make the incision in the outer wall of the duct and towards its posterior part, so that the escaping bile falls directly into the lowest part of the hepatic pouch, and before squeezing the stone out grasp either side of the incision in catch forceps as a guide later to the hole in the duct. When the stone has been removed, the common duct should be carefully examined, if possible, by a finger,and it generally is so dilated that this can be done,- and always with a long, thick probe. This should be passed first into the hepatic ducts and then into the duodenum, to ensure as far as possible that all stones have been taken away. Finally, the gall bladder should be opened and emptied. In these cases I drain the gall bladder by a tube sewn in, drain the common duct by a large tube sutured with catgut to the edges of the wound in the common duct, and the hepatic pouch by a large protective strand of gauze, all brought out at the most dependent part of the incision posteriorly.

The difficulties of the operation may be described as due to physiological and to pathological causes.

In the case of a short, stout patient, with active muscles, 
and a liver firmly tucked up under the ribs, who is difficult to anæsthetise satisfactorily, the operation is very much more difficult than in the class of patient previously mentioned.

The most serious difficulties are the result of pathological changes, which have occurred in the hepatic pouch. There all the structures may be found densely matted together by such strong adhesions, that injuries to the intestine below and to the liver above may be with every care unavoidable. Each injury must be noticed and repaired. The first landmark I wish to find in these difficult cases is the gall bladder. Once it has been isolated by drawing it downwards and forwards, the liver is fixed and pulled towards the surface, and the tense cystic duct guides the fingers to the common bile duct, and a stone or stones in it. These are all, if possible, removed from the ducts, the gall bladder is emptied, and drainage completes the operation.

It will be noted that I have said, if possible, all stones are removed; and this is an important qualification. From published records of gallstone operations, it might be assumed that it was always possible to remove the stones, but this assumption would be very far from the truth. In cases of long duration, where there are multiple stones in the common duct, the whole of the hepatic ducts are dilated and frequently contain stones. When they do, it has been my experience that all the stones are seldom removed, for the very good reason that it is impossible to reach them. I have abundant post-mortem evidence of the truth of this statement, for I have seen, on many occasions, stones in every part of the liver, both in the ducts and lying outside of them in abscess cavities. A perusal of the notes of my fatal cases will show that many of them were truly inoperable, and the fact should be recognised that delayed operations may do more harm than good. The difficulty is to recognise these unsuitable cases before exploring them; but when once the operation has been undertaken, it is needful to do all that can be done for their relief. If stones are discovered in the hepatic ducts, all that are easily accessible should of course be removed, but no prolonged or serious manipulations should be indulged in. Drainage of the gall bladder and ducts must be relied on to do the rest. In the most serious cases I incline to the view that to establish a fistula between the gall bladder and the intestine (cholecystenterostomy) is the proper treatment. Nature cures occasionally in this way, and the hint is worthy of further consideration.

I operated in the case of a man deeply jaundiced and with symptoms of gallstone, and felt a stone impacted in his common duct. There were also nodules of malignant disease on the convexity of his liver. A prolonged operation was useless; and, without touching the ducts or stone, I performed chole- 
cystenterostomy with a Murphy's button. The result exceeded my expectations, as for more than a year after the operation he was quite well; indeed, his recovery seemed to be so complete, till his symptoms recurred, that a distinguished surgeon was induced to remove the gall stone in the hope of curing him. It might have been better for some of the patients whose cases I am recording, if I had limited their operative treatment to cholecystenterostomy.

\section{NOTES ON TROPICAL DISEASES.}

By Major D. G. Marshall, I.M.S., Lecturer on Tropical Diseases in the School of Medicine of the Royal Colleges, Edinburgh.

The Treatment of Guinea-Worm.-As one of the speakers who took part in the discussion on guinea-worm at the recent meeting of the British Medical Association truly remarked-" the great amount of physical infirmity caused by this affection is not fully understood, and it would be well were the disease more carefully studied."

Little advance has been made in the treatment of guineaworm, most writers on the subject confining themselves to a description of the measures to be adopted when the worm is extruding. These measures vary from the ancient method of twisting out the worm on a piece of stick, to injection of corrosive sublimate, alcohol, etc.

There is, however, another and better form of procedure, namely, early detection and removal of the worm or worms while still free in the subcutaneous tissues, and before an attachment to the skin has been formed.

This method, as described below, should be followed not only when the presence of guinea-worm is suspected, but also when one is extruding. Multiple infection is very common, and in those cases where one worm is showing, careful examination will often reveal the presence of other worms in different parts of the body.

Detection.-The patient should be stripped, and the whole surface of the body examined. Occasionally the presence of a worm under the skin may be detected by inspection, but it is a good rule not to trust to inspection alone but to examine further by palpation-the whole skin surface being gently stroked with the fingers, - the presence of a worm, often in unexpected parts, as the arm, neck, or abdominal wall, imparting the feeling as though a piece of soft string was lying under the skin.

Removal.-This is a simple proceeding, no anæsthetic being usually required. The skin over the worm having been rapidly washed with a strong antiseptic, is stretched between two fingers of the left hand and divided by a small incision (about half an 